\title{
Adsorbed Cobalt Porphyrins Act like Metal Surfaces in Electrocatalysis
}

\author{
Corey J. Kaminsky, ${ }^{\dagger}$ Sophia Weng, ${ }^{\dagger}$ Joshua Wright, ${ }^{\star \S}$ and Yogesh Surendranath ${ }^{\dagger *}$ \\ ${ }^{\dagger}$ Department of Chemistry, Massachusetts Institute of Technology, Cambridge, Massachusetts 02139, United States \\ *Advanced Photon Source, Argonne National Laboratory, 9700 Cass Avenue, Lemont, Illinois 60439, United States \\ §Illinois Institute of Technology, 3300 South Federal Street, Chicago, Illinois 60616, United States
}

\section{Solvated:}

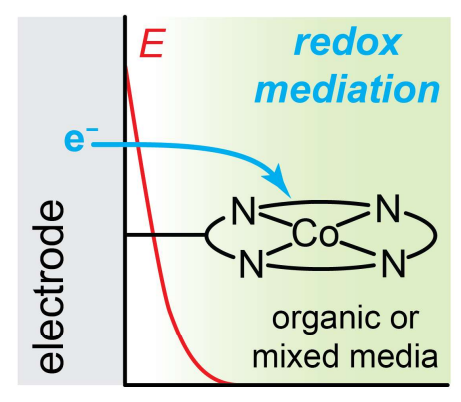

Operates like molecules
Adsorbed:

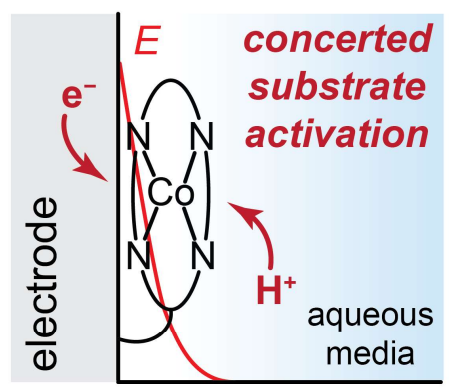

Operates like metal surfaces

\begin{abstract}
Carbon electrodes chemically modified with molecular active sites are potent catalysts for key energy conversion reactions. Generally, it is assumed that these molecularly modified electrodes operate by the same redox mediation mechanisms observed for soluble molecules, in which electron transfer and substrate activation occur in separate elementary steps. Here, we uncover that, depending on the solvent, carbon-bound cobalt porphyrin can carry out electrolysis by the non-mediated mechanisms of metal surfaces in which electron transfer and substrate activation are concerted. We chemically modify glassy carbon electrodes with cobalt tetraphenylporphyrin units that are anchored by flexible aliphatic linkages to form CH-CoTPP. In acetonitrile, CH-CoTPP displays a clear outer-sphere $\mathrm{Co}(\mathrm{II} / \mathrm{I})$ process which catalyzes the $\mathrm{H}_{2}$ evolution reaction by a step-wise, redox-mediated reaction sequence. In contrast, clear surface redox waves are not observed for $\mathrm{CH}$-CoTPP in aqueous media and $\mathrm{H}_{2}$ evolution proceeds via a non-mediated, concerted proton-electron transfer reaction sequence over a wide $\mathrm{pH}$ range. The data suggest that, in aqueous electrolyte, the CoTPP fragments reside inside the electrochemical double layer and are electrostatically coupled to the surface. This coupling allows CH-CoTPP to carry out catalysis without being pinned to the redox potential of the molecular fragment. These studies highlight that the simple adsorption of molecules can lead to reaction mechanisms typically reserved for metal surfaces, exposing new principles for the design of molecularly-modified electrodes.
\end{abstract}

\section{INTRODUCTION}

An economy based on renewable energy requires efficient catalysts for interconverting between chemical and electrical energy. Molecular catalysts can be appended to inert electrodes to generate chemically-modified electrodes (CMEs) that are potent catalysts for key reactions in next generation energy conversion devices. ${ }^{1-30}$ While there are many strategies to prepare CMEs, a widely employed and convenient approach is the simple adsorption of the molecular component onto the electrode surface. ${ }^{3-13,15,17,19,20,22-27}$ This ease of preparation along with the high degree of tunability afforded by the molecular component and overall potent reactivity make CMEs compelling catalyst materials. It is thus desirable to uncover the design principles for CMEs to enable rational optimization of this important class of catalysts. ${ }^{24,26-29}$

The design of CMEs usually centers on the optimization of the molecular catalyst because the electrode support is generally viewed as an inert source or sink of electrons. The synthesis of active CMEs thus follows two general steps: the selection of a known highly active molecular catalyst for the reaction of interest and the development of a heterogenization method. This synthetic logic assumes that activity trends in homogeneous molecular catalysts will correspond to the same trends in electrode activity upon heterogenization. However, there are flaws to these implicit assumptions: (a) for solubility reasons, most molecular electrocatalysts are evaluated and optimized in aprotic polar organic solvents such as acetonitrile and DMF with added proton donors. In contrast, catalysis by CMEs is typically evaluated in the aqueous media relevant to device operation, which has starkly different solvation properties and proton conductivities. both of which are expected to dramatically impact the mechanisms of the proton-coupled electron transfer reactions. Further, (b) in molecular electrocatalysis, substrate activation take place in solution, far from the enormous electric fields native to the electrode surface. ${ }^{32-39}$ Since electric fields impact 
the reactivity of enzymes, ${ }^{40-42}$ surfaces, ${ }^{32-39,43,44}$ and molecules, ${ }^{45-50}$ there is every reason to expect that they may influence reactivity of CMEs. ${ }^{28,32,38} \mathrm{An}$ improved understanding of the mechanistic implications of (a) and (b) has the potential to expose new design principles for the rational development of more active CMEs.

Recent observations lend credence to the notion that heterogenization does alter catalytic activity. ${ }^{24,26,27,29-32}$ For example immobilization of an iron porphyrin results in a 26 -fold rate enhancement versus the same species dissolved in the same reaction media. ${ }^{30}$ Other studies have shown that the reactivity of an appended catalyst is tuned by diverse factors such as immobilization method, ${ }^{24,31}$ electrode surface chemistry ${ }^{26,27}$ and polymer binder identity. ${ }^{16,27}$ Though these factors are as important as the catalyst structure in defining its reactivity, the origins of these effects remain unclear. ${ }^{16,24,26,27}$ In a poignant example, pyrazine linkages that engender strong electronic coupling between molecules and the surface prevent the immobilized catalyst from operating by the step-wise pathways characteristic of molecular catalysts and instead enforce the concerted mechanisms unique to heterogeneous metallic catalysts. ${ }^{29,51,52}$ Clearly, the rational development of CMEs requires greater understanding of the molecule-surface interactions that play a key role in defining their reactivity.

In this study, we directly probe the impact of strong surface interactions on catalysis by adsorbed molecules. Counterintuitively, instead of directly adsorbing the molecular species to the surface, we anchored a cobalt tetraphenylporphyrin (CoTPP) to an oxidized glassy carbon electrode with a covalent aliphatic linkage. We chose this linkage to minimize convolution from multilayer adsorption, ${ }^{53-57}$ prevent dynamic aggregation on the surface, ${ }^{22}$ and permit the comparison of reactivity across diverse reaction media while maintaining a constant surface population of active sites. Importantly, we chose a flexible cyclohexyl tether that allows the appended CoTPP to adopt either an adsorbed or solvated configuration, depending upon the solvating properties of the electrolyte. Thus, by examining catalytic trends across different reaction media, we isolate the critical role of surface interactions on catalysis by CMEs.

Herein, we identify that surface adsorption of CoTPP confers electrostatic coupling to the electrode surface with dramatic implications for reaction mechanism and catalyst design. We identify these results by comparing the hydrogen evolution reactivity (HER) of CoTPP covalently-linked to a glassy carbon electrode in acetonitrile, aqueous, and mixed aqueous-pyridine electrolytes. We further compare the surface-bound CoTPP to a water-soluble analog in aqueous electrolyte. We demonstrate that the step-wise reaction pathways observed for soluble molecules occur for this CME in media where CoTPP is soluble but that the concerted reaction pathways typical of metallic electrodes occur for this CME in media where CoTPP is insoluble. Finally, we provide a model to explain how this change in reaction mechanism could arise from strong adsorption to the surface. These results have broad implications for the design of CMEs and suggests that the design criteria for optimizing soluble molecular catalysts may not apply upon adsorption to the electrode surface.

\section{RESULTS AND DISCUSSION}

\section{Synthesis and Characterization}

The necessary surface functionalization precursor containing an insulating aliphatic linkage was accessed via treatment of 5( $p$-aminophenyl)-10,15,20-triphenylporphyrin with trans-4-(BOC-amino)cyclohexanecarboxoyl chloride followed by removal of the BOC group with trifluoroacetic acid to yield trans-4-amino-N-(4-(10,15,20-triphenylporphyrin-5-yl)phenyl)cyclohexane-1-carboxamide $\left(\mathrm{H}_{2} \mathrm{PorNH}_{2}\right)$. The cobalt-coordinated species, $\mathrm{CoPorNH} \mathrm{H}_{2}$ was accessed via treatment of $\mathrm{H}_{2} \mathrm{PorNH}_{2}$ with cobalt acetate tetrahydrate. Functionalization of the surface proceeded readily by modification of literature procedures. ${ }^{31}$, ${ }^{58-60}$ Specifically, surface acyl chlorides were generated by treatment of oxidized glassy carbon electrodes with thionyl chloride. Subsequent treatment of the electrodes with either $\mathrm{H}_{2} \mathrm{PorNH}_{2}$ or $\mathrm{CoPorNH}_{2}$ in the presence of pyridine generated $\mathrm{CH}-$ MTPP (M = 2H, Co) (Scheme 1). Full synthetic details are provided in the Supporting Information (SI).

\section{Scheme 1. Preparation of CH-MTPP electrodes}

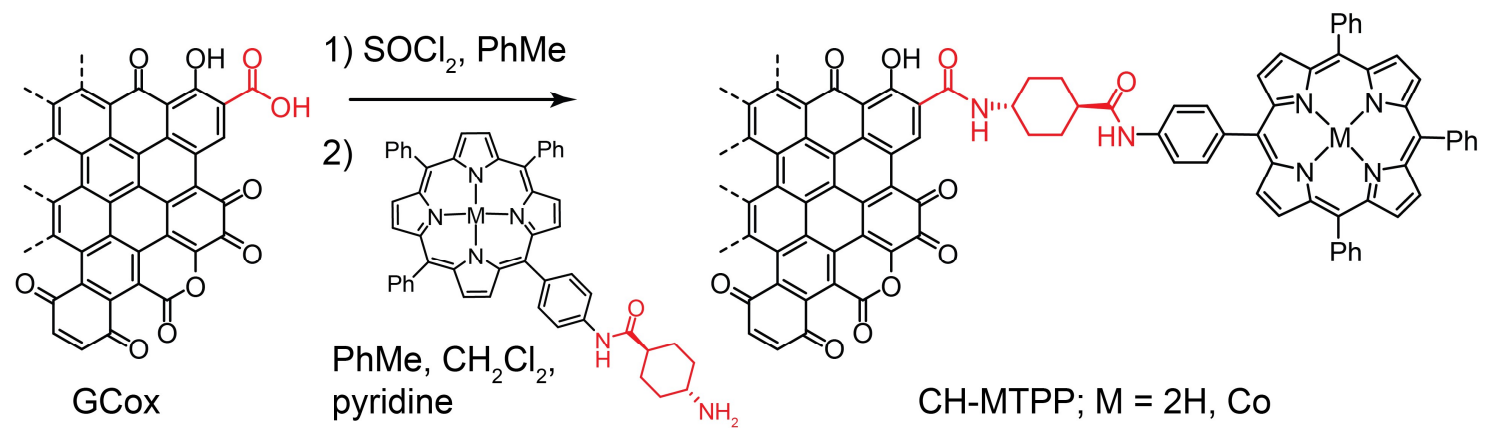


X-ray photoelectron spectroscopy (XPS) evinces successful ligation of the molecular precursor to the graphitic support to form CH-CoTPP. Following functionalization, new features corresponding to $\mathrm{N} 1 s$ and $\mathrm{Co} 2 p$ transitions appear in the survey analysis (Figure S1). High resolution scans of these features reveal an $\mathrm{N} 1 s$ peak manifold that is fit well with three peaks centered at 398.5, 399.7 and $401.4 \mathrm{eV}$ that we assign to porphyrin, ${ }^{31}$ amide, ${ }^{61}$ and pyridinium ${ }^{61,62}$ nitrogen environments, respectively (Figure 1a). The porphyrin and amide features integrate in the expected 2:1 ratio consistent with the structure of CH-CoTPP. The integration of the pyridinium feature varies from 1 to 1.5 with respect to the amide feature. The binding energy of this feature is inconsistent with metal-bound pyridinic nitrogen and structural data is most consistent with square planar cobalt (see below). Thus, we attribute this feature to the reaction of pyridine with reactive surface chlorides that result from the thionyl chloride treatment. Further, this feature is not observed or is greatly attenuated after electrochemistry in aqueous media where such species would be expected to hydrolyze (Figure S2). The high-resolution spectrum of the Co $2 p$ peak manifold reveals a single $2 p$ doublet at 779.9 and $795.1 \mathrm{eV}$, indicative of only a single cobalt environment on the surface (Figure 1b). The observed Co $2 p$ binding energies are consistent with literature reports on cobalt porphyrins. ${ }^{31,63}$ The relative integrations of the amide and porphyrin together against the Co $2 p$ peak manifold yields a N:Co ratio of 8 (expected $=6$ ), suggesting some demetallation occurs during the surface functionalization procedure. Together, the XPS data support the formation of the amide linkages and the presence of intact porphyrin units on the surface.

a)

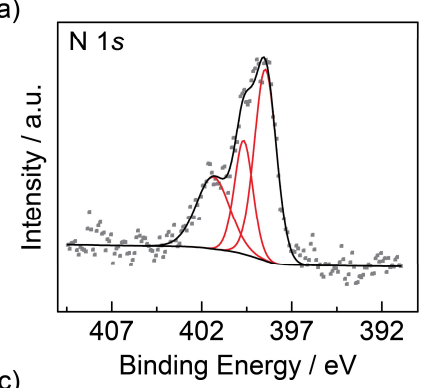

c)

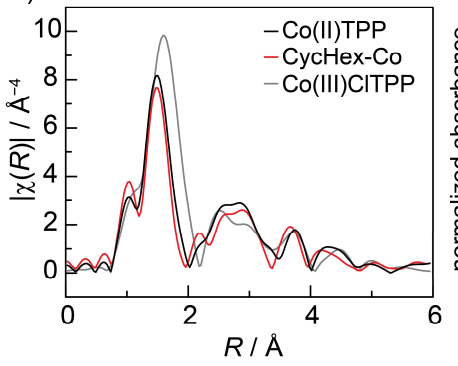

b)
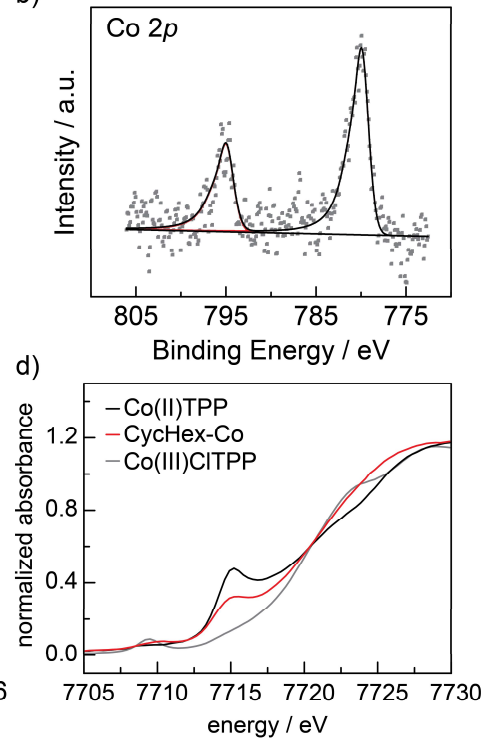

Figure 1. Characterization of CH-CoTPP. High-resolution XPS scans of the $\mathrm{N} 1 s$ (a) and Co $2 p$ (b) regions with the measured signal (gray points), fit peaks (red), background and overall fit peak envelope (black). c) Extended X-ray absorption fine structure of CH-CoTPP and model complexes. d) X-ray absorption near edge structure spectrum of CH-CoTPP and model complexes.

X-ray absorption spectroscopy (XAS) further supports surface modification with intact CoTPP units. Cobalt K-edge extended $\mathrm{X}$-ray absorption fine structure analysis (EXAFS) provides a fingerprint for the molecular structure about the cobalt center. EXAFS of CH-CoTPP is nearly identical to that of the molecular analog, CoTPP, but does not fit well to CoClTPP that bears an axial ligand on the Co center (Figure 1c). These data reveal a square planar coordination environment for CH-CoTPP. The $\mathrm{X}$-ray absorption near-edge structure (XANES) provides complimentary electronic structural information. Consistent with previously examined covalently grafted porphyrins on graphitic carbon, ${ }^{31}$ the XANES spectrum of CH-CoTPP exhibits preedge features consistent with both the $\mathrm{Co}$ (II) and $\mathrm{Co}$ (III) standards that may indicate mixed valency character of the Co center on the surface (Figure 1d). The origin of the changes to the pre-edge features remains undetermined. Together with the XPS data, the XAS data support ligation of intact CoTPP units to the glassy carbon surface.

\section{Outer-Sphere Electron Transfer is Observed in Acetonitrile}

In acetonitrile electrolyte, the cyclic voltammogram (CV) of CH-CoTPP exhibits a clear outer-sphere Co(II/I) feature, consistent with analogous systems. ${ }^{60}$ In $0.1 \mathrm{M}$ tetrabutylammonium hexafluorophosphate $\left(\mathrm{TBAPF}_{6}\right)$ in acetonitrile, this feature occurs at $-0.76 \mathrm{~V}$ vs decamethylferrocene $\left(\mathrm{Fc}^{*} / \mathrm{Fc}^{*+}\right)$ (Figure 2). The peak current density of this feature scales linearly with 
scan rate, consistent with a surface-bound species (Figure S3). Importantly, the charge passed in this feature correlates well to the amount of cobalt on the surface, as determined by inductively-coupled plasma mass spectrometry (see SI for details). We find a Co-to-electron ratio of $1.05 \pm 0.07$ which further supports our assignment of this feature as a $\mathrm{Co}(\mathrm{II} / \mathrm{I})$ redox process. The observation of this feature in electrolyte without strongly-coordinating anions suggests that it is not ion-coupled. Further, this feature exhibits no dependence on chloride concentration, indicating that the feature is not tied to ion coordination or dissociation (Figure S4). Together, the data support that, in acetonitrile media, CH-CoTPP exhibits outer-sphere electron transfer (ET) behavior.

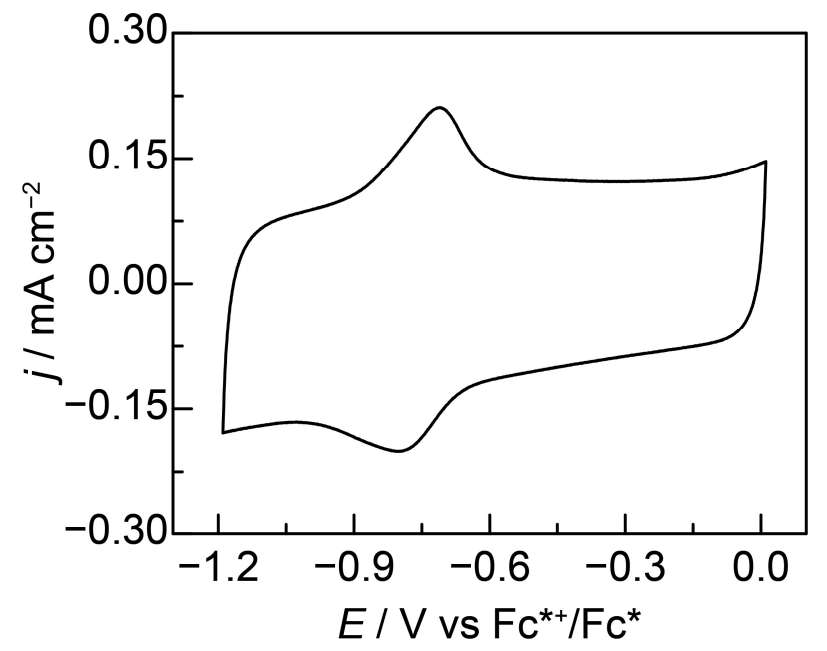

Figure 2. Cyclic voltammogram of CH-CoTPP in $0.1 \mathrm{M} \mathrm{TBAPF}_{6}$ in acetonitrile showing the outer-sphere $\mathrm{Co}(\mathrm{II} / \mathrm{I})$ redox couple. Data were recorded at $150 \mathrm{mV} \mathrm{s}^{-1}$ scan rate. The scan was initiated by sweeping reductively.

\section{Catalysis in Acetonitrile Displays Characteristics of Redox Mediation}

CH-CoTPP is a catalyst for HER in acetonitrile. $\mathrm{CVs}$ of $\mathrm{CH}-\mathrm{CoTPP}$ were collected in acetonitrile containing $0.1 \mathrm{M} \mathrm{TBAPF}_{6}$ and donors with defined proton activities. The proton activity was defined by addition of $25 \mathrm{mM}$ each of proton donor and conjugate base. In the presence of highly acidic donors, CH-CoTPP catalyzes the HER but decomposes too rapidly for in depth analysis. In the presence of a chloroacetic acid buffer $\left(\mathrm{p} K_{\mathrm{a}}=15.3 ; \mathrm{CH}_{2} \mathrm{ClCO}_{2} \mathrm{H}-\left[\mathrm{TBA}^{+}\right]\left[\mathrm{CH}_{2} \mathrm{ClCO}_{2}{ }^{-}\right]\right)^{64} \mathrm{CH}-\mathrm{CoTPP}$ catalyzes the HER with a near classical S-shaped wave observed at a scan rate of $5 \mathrm{mV} \mathrm{s}^{-1}$ (Figure 3a). The onset of this Sshape wave is tied to the Co(II/I) redox couple. Such behavior indicates mediated catalysis that operates by step-wise outersphere pathways. ${ }^{65,66}$ Charge integrations of the Co(II/I) redox wave with the faster scan rate CVs that beat out catalysis in the chloroacetic acid buffer accounts for $\sim 75 \%$ of the total loading of Co on the surface, indicating that CH-CoTPP does not substantially decompose under these reaction conditions (Figure S5).

To further support the assignment of a mediated step-wise mechanism, catalysis was evaluated in the presence of a more basic donor. Redox mediated reactions are pinned to an outer-sphere reduction potential but $E_{\mathrm{RHE}}$ shifts Nernstianly with the $\mathrm{p} K_{\mathrm{a}}$ of the proton donor ${ }^{67,68}$ For a step-wise reaction, selection of a sufficiently basic donor can move $E_{1 / 2}$ to an underpotential of $E_{\mathrm{RHE}}$ and catalysis will shut off. Such behavior is diagnostic of a redox mediated sequence proceeding by outer-sphere step-wise electron transfers. ${ }^{52} \mathrm{CVs}$ of CH-CoTPP were collected in the presence of an acetic acid buffer $\left(\mathrm{p} K_{\mathrm{a}}=22.3\right.$; AcOH$\left.\left[\mathrm{TBA}^{+}\right]\left[\mathrm{AcO}^{-}\right]\right),{ }^{67}$ where $E_{1 / 2}\left(\mathrm{Co}^{\mathrm{II} /}\right)$ lies at an estimated underpotential of $\sim 100 \mathrm{mV}$ (see SI). In the acetic acid buffer, no catalysis was observed off of the $\mathrm{Co}(\mathrm{II} / \mathrm{I})$ couple and the redox feature was recovered (Figure $3 \mathrm{~b}$ ). Importantly, the observation of clear $\mathrm{Co}(\mathrm{II} / \mathrm{I})$ waves in the acetic acid buffer demonstrates that CH-CoTPP does not decompose under these more basic conditions. Together, the observation of an S-shaped wave tied to the $\mathrm{Co}(\mathrm{II} / \mathrm{I})$ redox couple with chloroacetic acid buffer and the lack of catalysis with the more basic $\mathrm{AcOH}$ support a step-wise, redox mediated sequence initiated by outer-sphere electron transfer. 

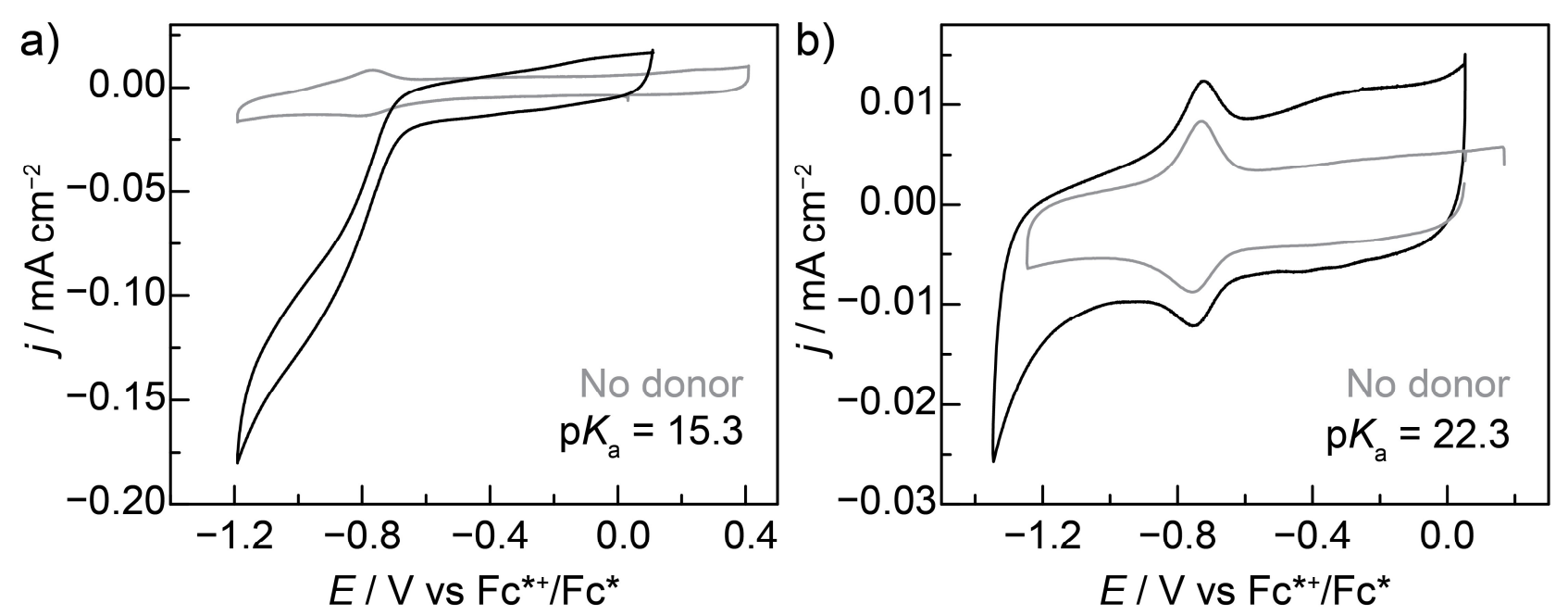

Figure 3. Cyclic voltammograms of CH-CoTPP in acetonitrile containing $0.1 \mathrm{M} \mathrm{TBAPF}_{6}$ (grey) and $25 \mathrm{mM}$ each of proton donor and conjugate base (black). Scans were recorded at $5 \mathrm{mV} \mathrm{s}^{-1}$. a) The donor and conjugate base are $\mathrm{CH}_{2} \mathrm{ClCO}_{2} \mathrm{H}$ and $\left[\mathrm{TBA}^{+}\right]\left[\mathrm{CH}_{2} \mathrm{ClCO}_{2}^{-}\right]$, respectively. d) The donor and conjugate base are $\mathrm{AcOH}$ and $\left[\mathrm{TBA}^{+}\right]\left[\mathrm{AcO}^{-}\right]$, respectively.

\section{Clear Electron Transfer Waves are Not Observed in Aqueous Media}

In contrast to its behavior in acetonitrile, $\mathrm{CH}$-CoTPP does not exhibit clear Co-based redox features in aqueous media. To aid interpretation of the aqueous electrochemistry of CH-CoTPP, we first examined the water-soluble cobalt meso-tetra $(p$ sulfonatophenyl)porphyrin chloride (CoClTSP). CVs of CoClTSP reveal an outer-sphere Co(II/I) couple at $-0.64 \mathrm{~V}$ vs NHE in both $0.1 \mathrm{M}$ sodium phosphate at pH 6.4 and in $0.1 \mathrm{M} \mathrm{NaOH}$ (Figures S6 and S7). In acidic media, CoClTSP mediates HER catalysis preventing observation of the $\mathrm{Co}(\mathrm{II} / \mathrm{I})$ wave (Figure S8). Consistent with our measured value for the $E_{1 / 2}\left(\mathrm{Co}^{\mathrm{II} / \mathrm{I}}\right)$ for CoCITSP, heterogenized cobalt protoporphyrin IX exhibits a Co(II/I) couple at $-0.6 \mathrm{~V}$ vs SHE when incorporated into a film on pyrolytic graphite. ${ }^{69}$

Unlike for the soluble molecular analog, voltammetry of CH-CoTPP does not reveal redox waves attributable to an outersphere $\mathrm{Co}(\mathrm{II} / \mathrm{I})$ redox process. The $\mathrm{CV}$ of $\mathrm{CH}-\mathrm{CoTPP}$ in $0.1 \mathrm{M} \mathrm{HClO}_{4}$ reveals one feature near $0.6 \mathrm{~V}$ vs NHE (Figure 4a) which is also observed in the CV of bare glassy carbon in this electrolyte (Figure S10). We attribute this feature to surface quinone PCET features or ion-intercalation processes native to oxidized glassy carbon. ${ }^{70}$ In $0.1 \mathrm{M}$ phosphate buffer at $\mathrm{pH}$ 6.4, this feature is not observed and the $\mathrm{CV}$ of CH-CoTPP is featureless within the solvent window (Figure $4 \mathrm{~b}$ ). Finally, in $0.1 \mathrm{M} \mathrm{NaOH}$, the $\mathrm{CV}$ of $\mathrm{CH}-\mathrm{CoTPP}$ displays a broad redox wave centered at $-0.75 \mathrm{~V}$ vs NHE (Figure $4 \mathrm{c}$ ). The magnitude of this wave is highly variable across electrode preparations and the integrated charge in this wave does not correlate with the Co surface loading. Additionally, this feature is also observed in the CVs of CH- $\mathrm{H}_{2} \mathrm{TPP}$ (Figure S10), thus we conclude that it is not associated with a $\mathrm{Co}(\mathrm{II} / \mathrm{I})$ redox process. Importantly, $\mathrm{CVs}$ of $\mathrm{CH}-\mathrm{CoTPP}$ in acetonitrile still show a pronounced $\mathrm{Co}(\mathrm{II} / \mathrm{I})$ wave after collection of this aqueous electrochemistry (Figures S11), indicating that the attached CH-CoTPP units remain intact even though they do not exhibit a clear Co(II/I) redox wave in water.

Interestingly, an outer-sphere redox process for $\mathrm{CH}-\mathrm{CoTPP}$ in aqueous media can be recovered in a mixed solvent system. Initially we hypothesized that axial coordination of the Co species could recover the Co(II/I) wave. In non-aqueous media, the introduction of pyridine is known to induce a Nernstian shift in the Co(II/I) wave of heterogenized Co porphyrins to more negative potentials, ${ }^{60}$ indicative of pyridine ligation to Co(II). Additionally, Co porphyrins have also been heterogenized via axial ligation to surface-bound pyridine units, ${ }^{71-73}$ suggesting that pyridine binds strongly to CoTPP in aqueous media. Thus, we collected CVs of CH-CoTPP in aqueous $0.1 \mathrm{M} \mathrm{NaOH}$ containing $0.1 \mathrm{M}$ pyridine (Figure S12). In this media, no new waves were observed. However, further addition of pyridine to a final concentration of $3 \mathrm{M}(\sim 13 \% \mathrm{v} / \mathrm{v})$ results in the appearance of a new redox feature (Figure $4 \mathrm{~d}$ ). The new redox feature is centered at $-0.91 \mathrm{~V}$ vs NHE and the charge integration accounts for ca. $90 \%$ of the surface Co. This potential is $0.27 \mathrm{~V}$ more negative than the $\mathrm{Co}(\mathrm{II} / \mathrm{I})$ potential of water soluble CoTSP, consistent with the more donating phenyl substituents and, perhaps, due to the interfacial solvation environment and/or pyridine coordination. Nonetheless, based on the similarity in potentials and the correspondence in charge integration value, we assign this feature as a $\mathrm{Co}(\mathrm{II} / \mathrm{I})$ redox process. Importantly, the concentration of pyridine necessary to recover this wave in aqueous media is also enough to sparingly solvate the parent CoTPP molecule (Figure S13). Given the literature precedence and the high concentration necessary to reveal this $\mathrm{Co}(\mathrm{II} / \mathrm{I})$ wave, it is likely that both the solvation of the CoTPP unit by pyridine and axial ligation are responsible for the change in the electrochemical response of CH-CoTPP. 

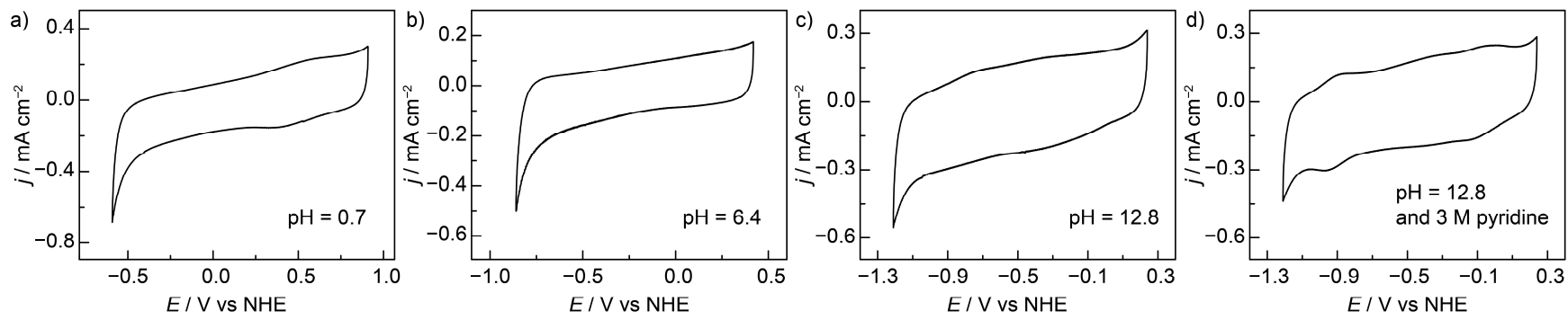

Figure 4. Cyclic voltammograms of CH-CoTPP in various aqueous buffers recorded at $100 \mathrm{mV} \mathrm{s}^{-1}$ in (a) $0.1 \mathrm{M} \mathrm{HClO}_{4}$, (b) $0.1 \mathrm{M}$ sodium phosphate buffer, (c) $0.1 \mathrm{M} \mathrm{NaOH}$ and d) $0.1 \mathrm{M} \mathrm{NaOH}$ with $3 \mathrm{M}$ pyridine. All scans were initiated by sweeping reductively.
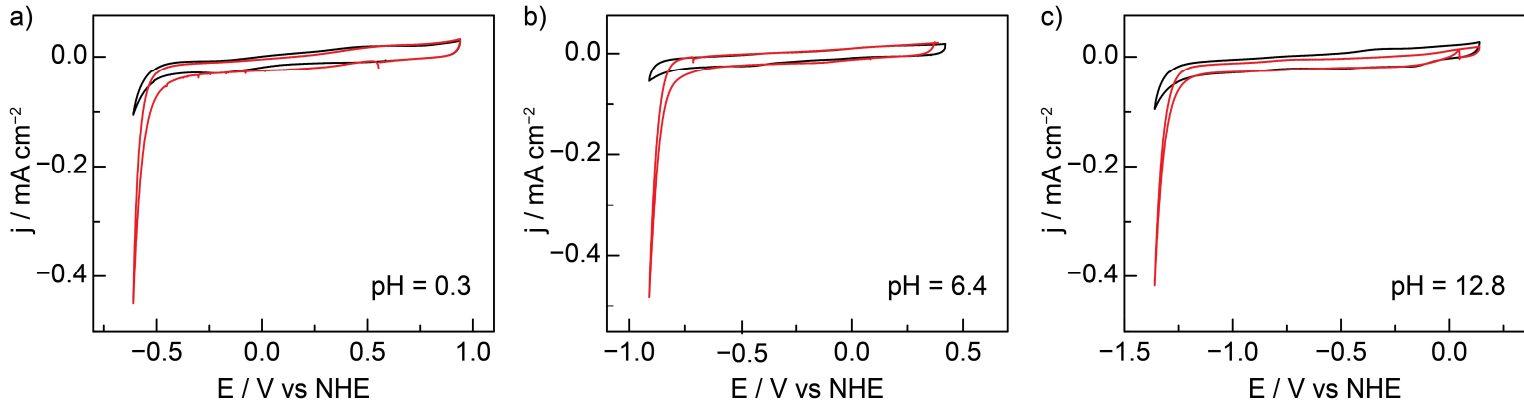

Figure 5. Cyclic voltammograms of HER catalysis by $\mathrm{CH}-\mathrm{CoTPP}$ (red) and $\mathrm{CH}-\mathrm{H}_{2} \mathrm{TPP}$ (black) in aqueous media. All scans were collected at $5 \mathrm{mV} \mathrm{s}^{-1}$ in (a) $0.1 \mathrm{M} \mathrm{HClO}_{4}$, (b) $0.1 \mathrm{M}$ sodium phosphate buffer, (c) $0.1 \mathrm{M} \mathrm{NaOH}$. All scans were recorded while the electrode was rotated at $2000 \mathrm{rpm}$. All scans were initiated by sweeping reductively.

\section{Catalysis Operates via Inner-Sphere Pathways in Aqueous Media}

In aqueous media, $\mathrm{CH}-\mathrm{CoTPP}$ shows Nernstian scaling for the onset of HER catalysis. In aqueous media over a $\mathrm{pH}$ range of 0.3 to 12.8 , CH-CoTPP (Figure 5, red) displays catalysis significantly above the background voltammograms of $\mathrm{CH}-\mathrm{H}_{2} \mathrm{TPP}$ (Figure 5, black). To investigate the mechanism of catalysis, we collected state-state current-voltage (Tafel) data in buffers across a wide $\mathrm{pH}$ range. Control experiments establish that these data are not convoluted by mass transport artifacts or deactivation on the time-scale of the measurement (Figures S14-S19). These data therefore correspond to activation-controlled rates for CH-CoTPP catalyzed HER. Though high-resolution XPS scans of CH-CoTPP after catalysis at each pH reveal minor amounts of demetallation during catalysis, particularly in acidic media, they show no peaks for $\mathrm{Co}^{0}$ (Figure S20). Since the post-catalysis XPS data indicates no detectable formation of Co nanoparticles during catalysis, we attribute the HER activity to intact CH-CoTPP.

Extraction of per-site TOFs of $1 \mathrm{~s}^{-1}$ from the Tafel data reveals that CH-CoTPP catalyzes HER with nearly constant overpotential across the $\mathrm{pH}$ range (Figure 6, red circles and both shaded regions). Indeed, the potential required to sustain a TOF of 1 $\mathrm{s}^{-1}$ shifts $58 \mathrm{mV}$ per $\mathrm{pH}$ unit (Figure 6, red dashed line), in line with the Nernstian scaling of $59 \mathrm{mV}$ per $\mathrm{pH}$ unit of $E_{\text {RHE }}$ (Figure 6 , solid black line). This shift is inconsistent with a redox-mediated step-wise reaction sequence for HER and suggests that a non-mediated, concerted sequence may be operative for CH-CoTPP. ${ }^{52}$ Redox mediated catalysis is pinned to the $E_{1 / 2}$ of the mediator redox couple; $; 5,66$ if a redox mediation sequence were operative for CH-CoTPP, the span of potentials over which $\mathrm{CH}-\mathrm{CoTPP}$ catalyzes HER in water indicates that the hypothetical $E_{1 / 2}\left(\mathrm{Co}^{\mathrm{II} / \mathrm{I}}\right)$ must lie more negative than $-1.4 \mathrm{~V}$ vs NHE. This upper bound value is $0.8 \mathrm{~V}$ more negative than the $E_{1 / 2}\left(\mathrm{Co}^{\mathrm{II} / \mathrm{I}}\right)$ value of $-0.64 \mathrm{~V}$ vs NHE for the water-soluble CoClTSP analog and $0.5 \mathrm{~V}$ more negative than $E_{1 / 2}\left(\mathrm{Co}^{\mathrm{II} / \mathrm{I}}\right)$ value of $-0.91 \mathrm{~V}$ vs NHE for CH-CoTPP in $0.1 \mathrm{M} \mathrm{NaOH}$ containing $3 \mathrm{M}$ pyridine. While CH-CoTPP catalyzes HER with a comparable overpotential across the full $\mathrm{pH}$ range, the water soluble CoClTSP only mediates HER off of $E_{1 / 2}\left(\mathrm{Co}^{\mathrm{II} / \mathrm{I}}\right)$ in acidic aqueous media (Figure 6, blue shaded region). Instead, $\mathrm{Co}(\mathrm{II} / \mathrm{I})$ waves are observed in neutral and basic media where there is insufficient driving force for the $\mathrm{Co}(\mathrm{II} / \mathrm{I})$ couple of CoClTSP to catalyze HER (Figure 6, blue squares). This starkly different $\mathrm{pH}$ scaling behavior between $\mathrm{CH}-\mathrm{CoTPP}$ and CoClTSP in aqueous media further suggests that CH-CoTPP does not operate by mediated sequences. While we acknowledge that CH-CoTPP could mediate HER, in principle, via different redox couples at varying $\mathrm{pH}$ values, the transition between these distinct mediating couples is unlikely to give rise to constant overpotential for catalysis. Furthermore, in this limit, we would expect to observe outersphere ET waves for the preceding redox couples that are unable to mediate catalysis in more basic media, yet we do not observe any clear redox waves for CH-CoTPP in aqueous media without addition of a large concentration of pyridine. Based 
on the forgoing analysis, the observation that CH-CoTPP catalyzes HER with similar overpotential across the pH range argues against a classical mediated redox sequence in water.

To gain further insight into the mechanism of HER catalysis by CH-CoTPP, we analyzed the Tafel slope and measured theH/D isotope effect for HER. In aqueous $0.5 \mathrm{M} \mathrm{HClO}_{4}$ and $0.1 \mathrm{M} \mathrm{NaOH}, \mathrm{CH}-\mathrm{CoTPP}$ displays Tafel slopes of 110 and $103 \mathrm{mV} \mathrm{dec}^{-1}$, respectively. These values correspond to transfer coefficients of 0.5 and 0.6 , which are consistent with a rate-limiting charge transfer step (Figures 7, top, and S18) ${ }^{74,75}$ Control experiments demonstrate no significant deactivation of the catalyst during these measurements and that these values are not convoluted by mass transport limitations (Figures 7, top, and S20). Importantly, these values are inconsistent with mechanisms invoking pre-equilibrium reduction of the Co center prior to ratelimiting proton transfer, which would yield a transfer coefficient of 1.0 and a Tafel slope of $59 \mathrm{mV} \mathrm{dec}{ }^{-1}$. ${ }^{74-76}$ Additionally, comparison of HER data collected in $0.5 \mathrm{M} \mathrm{HClO}_{4}$ in $\mathrm{H}_{2} \mathrm{O}$ and $\mathrm{D}_{2} \mathrm{O}$ reveals an $\mathrm{H} / \mathrm{D}$ isotope effect of $2.9 \pm 0.1$, indicative of a proton transfer involved in the rate-limiting step (Figures 7, bottom, and S21). Together, the Tafel and H/D isotope data are consistent with an HER mechanism by CH-CoTPP involving rate-limiting concerted proton-electron transfer (CPET). Though CPET reactions of water-soluble small molecules and coordination compounds can be driven by outer-sphere ET from an electrode, these mechanisms are generally restricted to a relatively narrow $\mathrm{pH}$ or potential range over which both possible stepwise pathways are energetically disfavored. ${ }^{65,77-80}$ Thus, the Tafel and H/D isotope effect data, taken together with the observation of Nernstian scaling and similar mechanistic profiles across the entire $\mathrm{pH}$ range leads us to invoke an inner-sphere, non-mediated concerted mechanism for HER by CH-CoTPP in water.
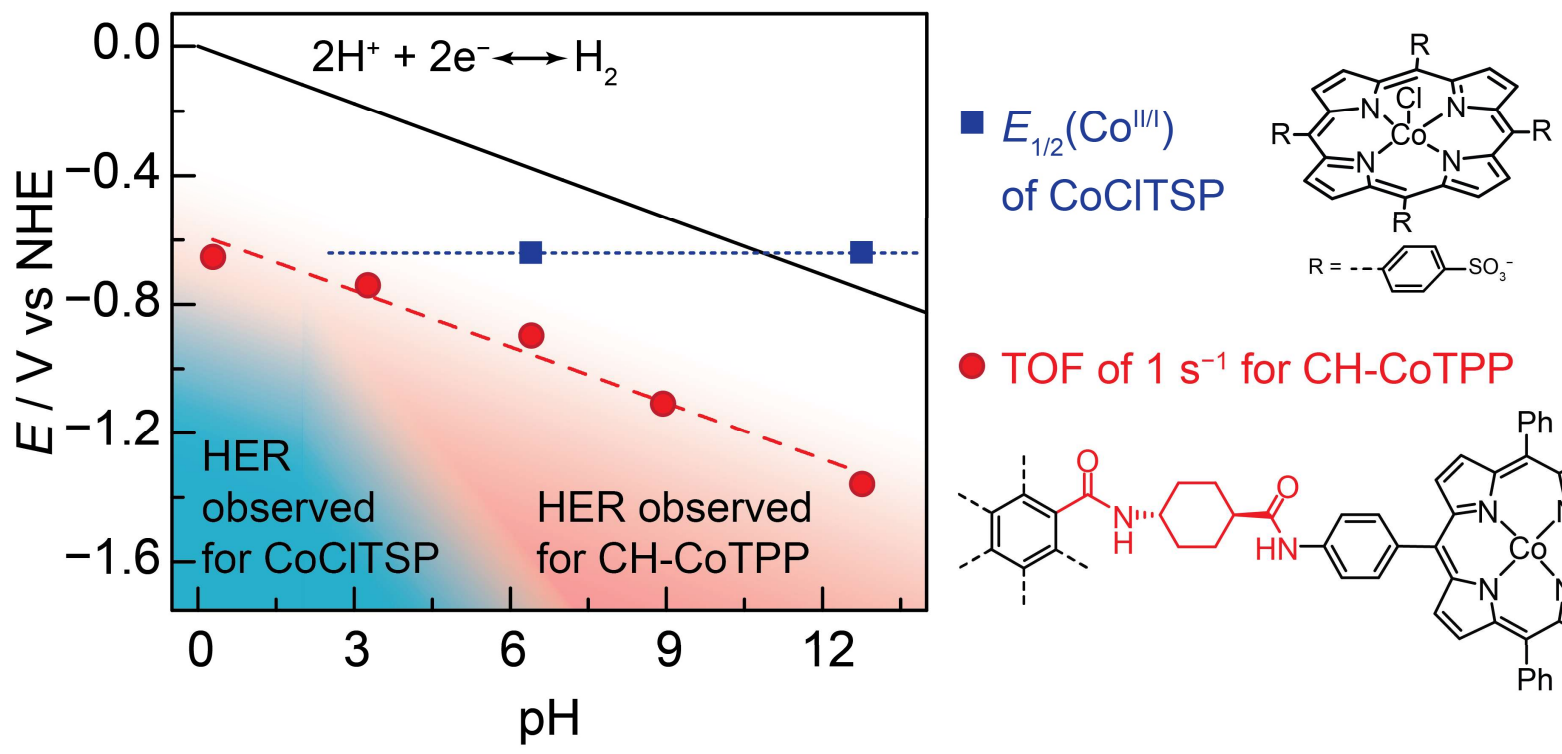

TOF of $1 \mathrm{~s}^{-1}$ for $\mathrm{CH}-\mathrm{CoTPP}$

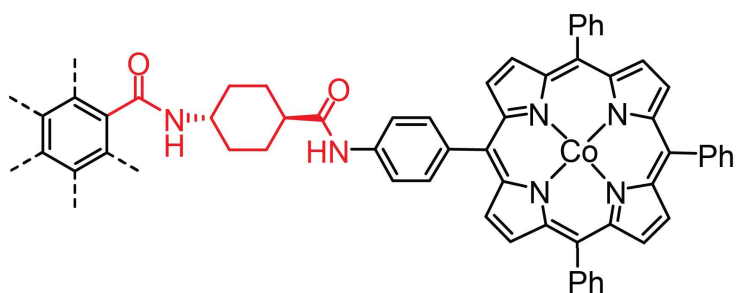

Figure 6. Potential vs pH (Pourbaix) diagram for CH-CoTPP and CoClTSP in aqueous electrolytes. The solid black line is the thermodynamic potential for HER in water. The red circles mark per-site turnover frequencies of $1 \mathrm{~s}^{-1}$ for CH-CoTPP with a linear best fit marked by a red dashed line with a slope of $58 \mathrm{mV}$ per $\mathrm{pH}$ unit. The blue squares mark $E_{1 / 2}\left(\mathrm{Co}^{\mathrm{II} / \mathrm{I}}\right)$ for the soluble CoCITSP with the blue dotted line to guide the eye to extrapolated values for this redox couple. The blue region represents where the $\mathrm{Co}(\mathrm{II} / \mathrm{I})$ couple of CoClTSP catalyzes HER while the red and blue regions together mark where CH-CoTPP catalyzes HER. 

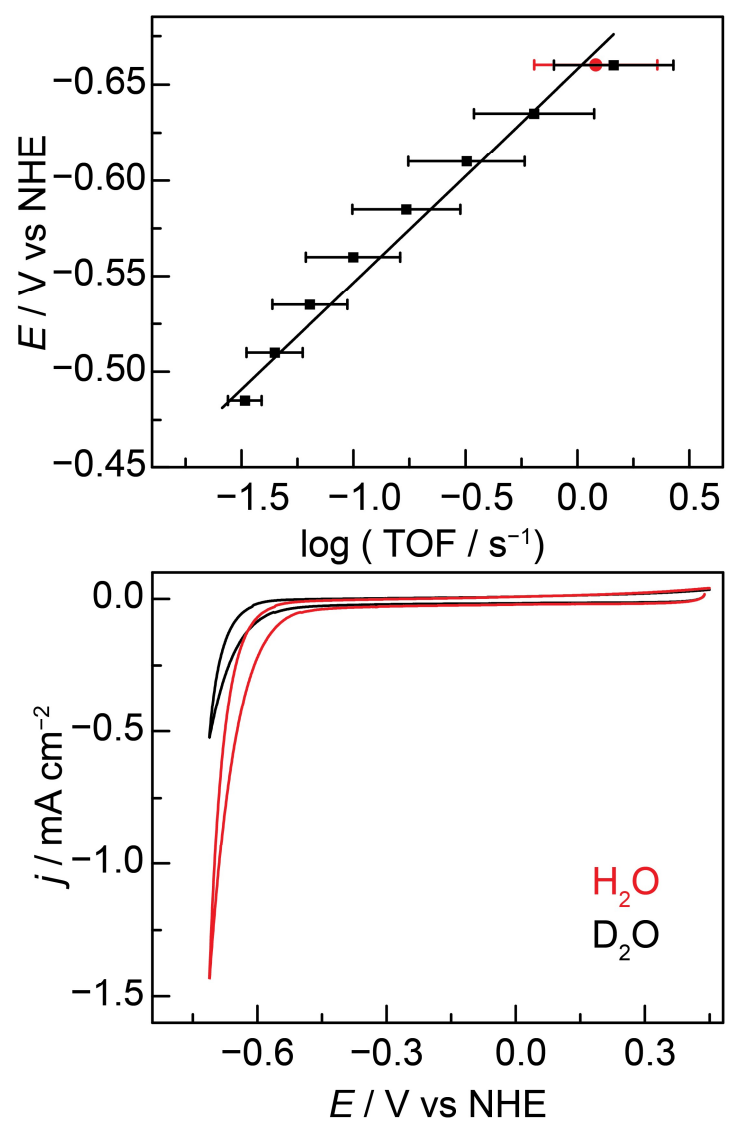

Figure 7. Kinetic data for HER by CH-CoTPP in aqueous acidic media. Top: Potential vs activation-controlled rate (Tafel) plot averaged over two measurements. The Tafel slope is $110 \mathrm{mV} \mathrm{dec}{ }^{-1}$. The error bars represent the standard deviation of two measurements. Data were collected from higher to lower overpotential, followed by recollection at the most negative applied potential to generate the red point. The similarity between the first point collected and the red point indicate that there is minimal change in activity over the course of data collection. Bottom: Cyclic voltammograms of the same CH-CoTPP electrode in $0.5 \mathrm{M} \mathrm{HClO}_{4}$ in $\mathrm{H}_{2} \mathrm{O}$ (red) and $\mathrm{D}_{2} \mathrm{O}$ (black) recorded with a scan rate of $25 \mathrm{mV} / \mathrm{s}$. For both panels, the data were measured while the electrode was rotated at $2000 \mathrm{rpm}$.

\section{Mechanistic Model}

The foregoing electrochemical data indicates distinct ET behaviors and HER mechanisms for CH-CoTPP in aqueous and acetonitrile electrolyte. In acetonitrile, we observe a clear outer-sphere $\mathrm{Co}(\mathrm{II} / \mathrm{I})$ redox process that is in quantitative agreement with the total cobalt population on the surface. This $\mathrm{Co}(\mathrm{II} / \mathrm{I})$ couple mediates HER catalysis and the $\mathrm{p} K_{\mathrm{a}}$-dependence supports a step-wise mechanism initiated by outer-sphere reduction of the cobalt center. In water, clear Co-based surface redox waves are not observed but are recovered upon addition of pyridine. Investigation of the mechanism of HER catalysis by CH-CoTPP in aqueous media supports a non-mediated mechanism in which proton transfer and electron flow are concerted. Additionally, we find that a water-soluble homogenous Co-TPP analog operates via step-wise HER pathways in aqueous media, suggesting that the change in mechanism for $\mathrm{CH}-\mathrm{CoTPP}$ requires surface interactions rather than simply resulting from the change int he reaction medium.

The non-mediated, concerted mechanism we observe for CH-CoTPP in aqueous media historically has been attributed solely to metal surfaces and recently has been implicated for graphite-conjugated catalysts (GCCs), which are molecules conjugated to carbon through aromatic linkages. The ET behavior of CH-CoTPP thus parallels the differences previously observed for GCCs and their soluble analogs ${ }^{29,31,51,52,81-83}$ but with the key distinction that the same surface-bound site displays mediated and non-mediated reaction mechanism depending on the electrolyte environment. Given the parallels to GCCs, we rationalize the solvent dependent behavior of CH-CoTPP by adapting the model put forth for GCCs. ${ }^{29,51,52}$ Specifically, we incorporate differential interactions with the electrode as a function of the electrolyte to explain the behavior of CH-CoTPP. Prior to discussing the specific surface interactions that might be at play, we discuss how the electrochemical behavior of CH-CoTPP in acetonitrile and aqueous electrolytes can be rationalized by distinct positioning of the Co center within the electrochemical double layer. A cartoon schematic energy diagram of the double layer is presented in Figure 8. In each panel, the rectangle represents the band states of the electrode, with filled states in grey and the Fermi energy, $E_{\mathrm{F}}$, marked in black. The position of $E_{\mathrm{F}}$ is sensed and directly modulated by the potentiostat during polarization. The red lines correspond to the electrostatic 
potential drop between $E_{\mathrm{F}}$ and the solution. The potential of zero free charge, $E_{\mathrm{PZFC}}$, is the potential at which there is no electrostatic potential drop between the electrode and solution. ${ }^{84}$ In the top panel, we denote a situation in which the Co center does not interact with the electrode and is instead freely solvated, which places it outside of the double layer electrostatic potential drop (red line). ${ }^{85}$ Alternatively, in the middle panel, we depict a situation in which the Co center is not freely solvated, but is adsorbed to the surface sufficiently strongly to reside within the electrochemical double layer (EDL). ${ }^{85}$

$\mathrm{MeCN}$
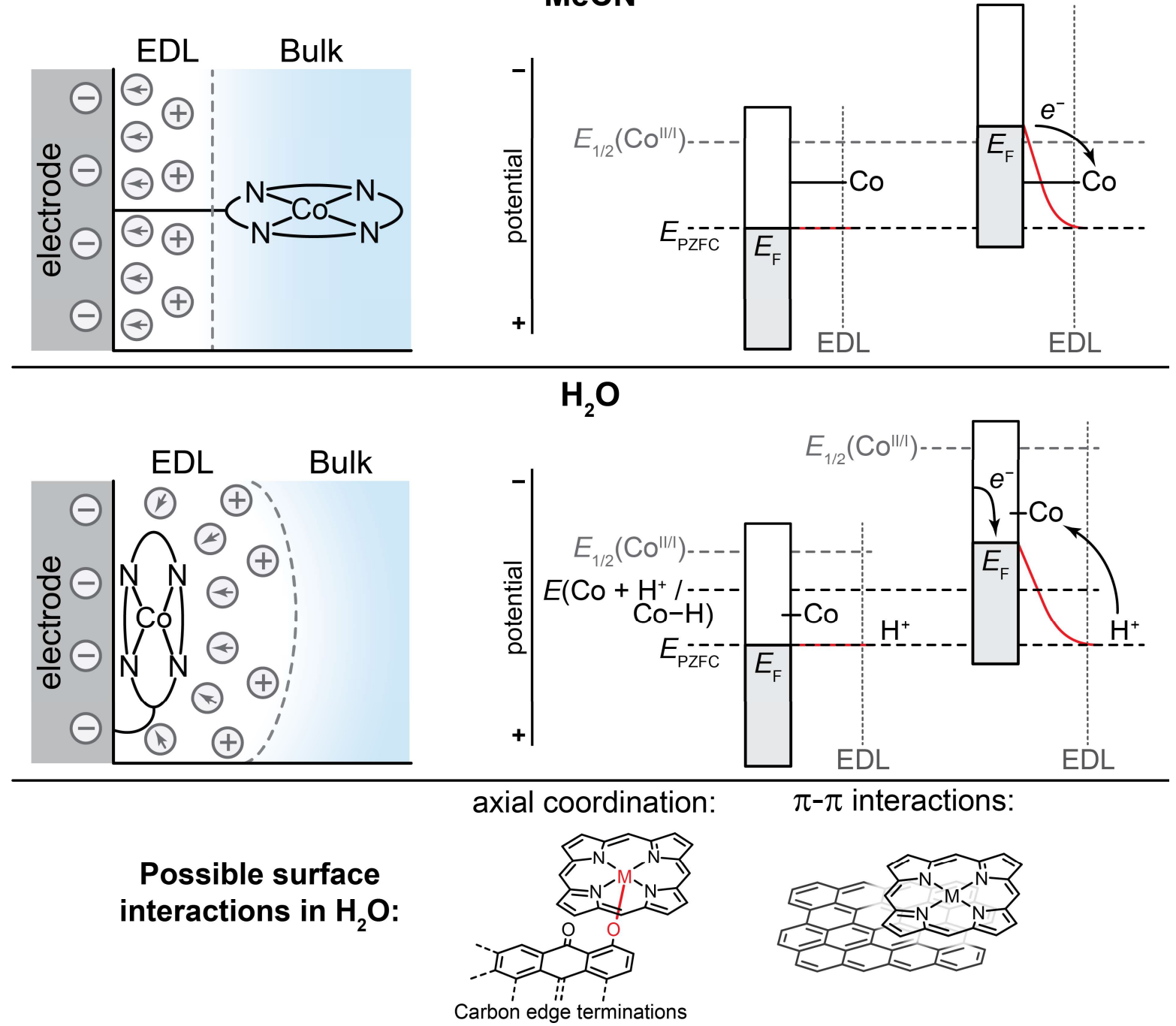

Figure 8. Cartoon models for the ET behavior of CH-CoTPP in acetonitrile (top) and water (middle) and the possible surface interactions that may stabilize the CoTPP unit within the EDL in aqueous media (bottom). The left part of each panel depicts the placement of the bound CoTPP unit with respect to the solvent and ion layers which comprise the EDL (white background) and the bulk solution (blue background) in acetonitrile (top) and water (middle). The right part of each panel depicts how polarization and relative placement with respect to the electrochemical double layer (EDL) leads to distinct electron transfer mechanism. The rectangles represent the density of states for the electrode, with the filled levels in grey and the black line between filled and unfilled is the Fermi level of the electrode, $E_{\mathrm{F}}$. The approximate edge of the EDL is marked with a vertical dotted grey line. The reduction potential of the Co center, $E_{1 / 2}\left(\mathrm{Co}^{\mathrm{I} / /}\right)$, and the potential for formation of the $\mathrm{Co}-\mathrm{H}$ intermediate are shown in dashed grey and black lines, respectively. The electrostatic potential drop at the interface is marked in red and the potential of zero free charge, $E_{\mathrm{PZFC}}$, where the electrostatic potential drop vanishes is marked with a dashed black line. The diagram indicates the relative positions of the aforementioned potentials upon polarization of the electrode (left to right). In acetonitrile, the model invokes that the Co center lies outside the EDL and polarization of the electrode drives outer-sphere electron transfer from the electrode to the CoTPP unit. In aqueous media, the model invokes that the Co center lies within the EDL and the Co energy levels are polarized in tandem with those of the electrode. The electrostatic potential drop drives proton transfer to the Co center with compensatory electron transfer from the external circuit to the electrode band states.

The data for the CH-CoTPP in acetonitrile points to the model shown in the top panel of Figure 8. This model details the classical behavior expected for dissolved molecules and molecules appended to but not interacting with an electrode ${ }^{86}$ In the top center, the electrode is polarized at $E_{\mathrm{PZFC}}$, where $E_{\mathrm{F}}$ lies at an underpotential of $E_{1 / 2}\left(\mathrm{Co}^{\mathrm{II} /}\right)$ and no ET occurs. Polarization 
of the electrode generates an interfacial electric field and raises the electronic levels of the electrode relative to those of the solution outside the EDL which are fixed. When $E_{\mathrm{F}}$ approaches or shifts more negative than the fixed $E_{1 / 2}\left(\mathrm{Co}^{\mathrm{II} / \mathrm{I}}\right)$, electrons from the electrode tunnel across the EDL to the CoTPP unit and more electrons are provided by the external circuit to fill the resulting holes in the electrode band states (right panel). ${ }^{84}$ This is exactly the behavior observed for dissolved molecules which lie outside the EDL. ${ }^{29,86}$ In acetonitrile, CH-CoTPP exhibits a clear outer-sphere Co(II/I) redox process (Figure 2) that is not proton-coupled and is consistent with the ET behavior shown in the top panel of Figure 8. Further, the S-shaped catalytic wave tied to the $\mathrm{Co}\left(\mathrm{II} / \mathrm{I}\right.$ ) redox couple and the $\mathrm{p} K_{\mathrm{a}}$-dependent changes to HER activity (Figure 3 ) indicate that the $\mathrm{Co}^{\mathrm{II} / \mathrm{I}}$ redox couple mediates catalysis. This imposes a rate-overpotential scaling relationship ${ }^{29,87-93}$ for HER activity by CH-CoTPP that causes catalysis to turn off under conditions in which $E_{\mathrm{RHE}}$ is close to or more negative of $E_{1 / 2}\left(\mathrm{Co}^{\mathrm{II} / \mathrm{I}}\right)$. Importantly, applying a more negative potential cannot recover catalytic activity by CH-CoTPP in the presence of strongly basic donors because the free energy of the reaction of $\mathrm{Co}(\mathrm{I})$ with proton is pinned by $E_{1 / 2}\left(\mathrm{Co}^{\mathrm{II} / \mathrm{I}}\right)$, regardless of the applied potential. Together, the data indicate that the energy levels of the Co center are well isolated from those of the electrode in acetonitrile and that ET proceeds by the tunneling of electrons across the EDL in direct analogy to the ET mechanisms observed for dissolved molecules. Thus, the model in which the Co center in CH-CoTPP resides outside the EDL is consistent with the experimental data in acetonitrile and implies that the Co center behaves like a dissolved molecule in this medium.

In aqueous media, the data for CH-CoTPP points to the model shown in the middle panel of Figure 8. Surprisingly, this is the same model that is invoked for metallic surfaces, not molecular species. Here, any shifts in $E_{\mathrm{F}}$ also result in tandem movements of the energy levels of the CoTPP unit. As a result, there is insufficient driving force for outer-sphere ET from the electrode to the Co center. Since this redox process is inaccessible, any mediated reaction pathways that proceed via outer-sphere ET steps are excluded. However, catalysis still occurs via inner-sphere mechanisms because there is a potential drop between the Co center and the solution. Instead of the tunneling of an electron, polarization drives the movement of an ion across the EDL to subsequently bind to the Co center. Thus, unlike the redox mediated mechanism described above, varying the strength of the proton donor across the $\mathrm{pH}$ range can be compensated for by polarization of the Co center to a more negative potential, thereby maintaining HER catalysis across the entire $\mathrm{pH}$ range (Figure 6). The charge of the proton brought to the Co center is compensated by concerted electron flow from the external circuit to maintain the electrode potential. This rate-limiting CPET sequence is consistent with the observed Tafel behavior and H/D isotope effect (Figure 7). Although charge-balancing the adsorption of one proton is expected to result in only a single ET from the external circuit, we still invoke formation of a metal hydride intermediate, $\mathrm{Co}-\mathrm{H}$. In our model, this two-electron bond arises from rapid internal rearrangement of electron density from the band states of the electrode ${ }^{94}$ rather than from a second ET from the external circuit. The catalytic cycle is completed when a soluble donor protonates this $\mathrm{Co}-\mathrm{H}$ intermediate and an electron flows from the circuit to balance the charge of the proton that crossed the EDL, to result in the overall two-electron stoichiometry of the HER. This model (Figure 8, middle) in which the Co center in CH-CoTPP resides inside the EDL is consistent with the Tafel, H/D isotope effect, and $\mathrm{pH}$ dependence data in water and requires that the CoTPP unit is interacting strongly with the electrode in this medium.

We attribute the starkly divergent ET and catalytic behavior in the above model to distinct positions of the CoTPP unit with respect to the EDL in each medium. This position defines whether there is a substantial electrostatic potential drop between the Co actives sites and the electrode (Figure 8, top) or not (Figure 8, middle). In acetonitrile, the data indicate that there are sufficient layers of solvent and ions between the surface and the CoTPP units to screen the Co centers from the charge on the electrode (Figure 8, top left). These intervening layers comprise the EDL and the majority of the electrostatic potential drop occurs across these solvent and ion layers. ${ }^{85}$ This model implies, that in acetonitrile, the relative free-energy of solvation of the molecular fragment and affinity of the solvent for the charged surface, outcompete the free energy of adsorption of the molecule to the surface..$^{95}$ In line with this reasoning, the parent CoTPP complex is sparingly soluble in acetonitrile (Figure S13) and $\mathrm{Co}(\mathrm{II} / \mathrm{I})$ waves can be recovered in aqueous media upon addition of $3 \mathrm{M}$ pyridine (Figure $5 \mathrm{~d}$ ), which sparingly solvates CoTPP (Figure S13).

In contrast, the aqueous data indicate that the catalytically active CoTPP units are not screened from the electrode charge in aqueous electrolyte and that there is no substantial electrostatic potential drop between the electrode and the Co centers. This lack of screening implies that there are insufficient layers of solvent and ions located between the electrode and the CoTPP units over the entire aqueous $\mathrm{pH} /$ potential window examined for HER (Figure 8, middle left). The majority of the electrostatic potential drop that occurs in the EDL thus lies between the Co active sites and bulk solution. In short, the active CoTPP units are cosolvated with the electrode. In line with this reasoning, we note that the parent CoTPP complex is insoluble in water (Figure S13). We posit that interactions with the electrode surface, such as $\pi-\pi$ interactions and/or axial coordination of the Co centers to oxidic edge terminations stabilize the CoTPP fragment within the EDL (Figure 8, bottom). It remains uncertain if these interactions confer a significant degree of quantum mechanical electronic coupling between the Co centers and the electrode band states, such as might be expected to a greater degree for the aromatic connectivity of GCCs. ${ }^{29}$ However, these interactions, at the very least, result in strong electrostatic coupling of the Co centers to the surface by exclusion of any intervening dielectric layers. Given the generality of these surface interactions for adsorbed porphyrins and phthalocyanines on graphitic carbons, the observation of non-mediated pathways for porphyrins heterogenized with alkyl tethers motivates a reevaluation of the reaction mechanisms of a wide variety of electrochemical transformations by heterogenized macrocycles.

\section{Conclusion}


We report the ligation of CoTPP to a graphitic carbon electrode through an aliphatic tether and examined its ET and catalytic behavior in acetonitrile and aqueous electrolytes. We show that the ET behavior follows a typical outer-sphere, redox-mediated, step-wise mechanism for HER in acetonitrile but transitions to a non-mediated concerted reaction pathway in aqueous electrolytes. This concerted mechanism by CH-CoTPP in aqueous media is akin to those of metallic electrode surfaces and bypasses the redox intermediates that pin the reactivity of molecular electrocatalysts. The starkly disparate reaction mechanisms in the two media are attributed to the different solvating properties of acetonitrile and water for the CoTPP unit. The preference for solvation over surface adsorption of the porphyrin in acetonitrile causes it to reside outside the EDL, whereas poor solvation in water favors strong adsorption to the surface, causing the molecule to reside inside the EDL. Thus, in aqueous media, preferential surface interactions lead to strong electrostatic coupling of the CoTPP units to the electrode which, in turn, drives inner-sphere, concerted reaction mechanisms.

Importantly, our model invokes nothing special about the covalent aliphatic amide linkage. While this covalent, flexible anchor was critical for us to expose distinct reaction mechanisms in aqueous and non-aqueous media, there is expected to be negligible coupling through this aliphatic tether. Thus, the linkage is not expected to contribute to the strong surface interactions that give rise to non-mediated concerted reaction mechanisms in water. Instead, the flexibility of the linkage permits other surface interactions to occur, if favorable. It is these other surface interactions that give rise to inner-sphere mechanisms in water and these native surface interactions are likely to exist for a wide variety of metallomacrocycle/carbon composite electrodes. Thus, these results motivate a reexamination of the electrochemical reaction mechanisms of adsorbed planar macrocycles. Specifically, these findings suggest that: (a) CMEs should not be presumed, a priori, to operate via step-wise mechanisms initiated by outer-sphere ET; (b) the surface redox waves observed for CMEs may correspond only to a fraction of the surface loading and catalytic activity may not arise solely from those "electroactive" surface sites; and (c) molecular fragments with redox potentials misplaced relative to the thermodynamic potential of the target reaction, may nonetheless be active as CMEs under electrolyte conditions that foster strong interactions with the electrode surface. Point (c) suggests that $E_{1 / 2}$ is not as useful a descriptor for identifying the molecular constituents of high-performance CMEs, particularly for molecules that are expected to adsorb strongly to electrode surfaces. Given that the adsorbed porphyrin examined here displays reactivity akin to bulk metal electrode surfaces, we posit that same descriptors used to optimize heterogeneous catalysts, ${ }^{96-98}$ such as the binding energies of key intermediates, may also be valuable in identifying new candidate molecules for high performance CMEs. Given that the concerted reactivity observed here circumvents the redox intermediates endemic to mediated catalysis, our findings open the door to a significant expansion of the use of strong molecule/surface interactions to enhance electrocatalysis on chemical modified electrodes.

\section{Data Availability}

The authors declare that the data supporting the findings of this study are available within the manuscript and Supplementary information files. Supplementary Information includes experimental procedures, post-mortem XPS and electrochemical data and extended catalysis data.

\section{AUTHOR INFORMATION}

Corresponding Author

*yogi@mit.edu

\section{Author Contributions}

C.J.K. and Y.S. conceived the research and developed experiments. C.J.K. and S.W. conducted all experiments. J.W. processed and analyzed X-ray absorption data. C.J.K. and Y.S wrote the manuscript with input from all authors.

Present Address

C.J.K.: Lawrence Berkeley National Laboratory, 1 Cyclotron Rd., Berkeley, CA 94720

Competing Interests

The authors declare no competing financial interest.

\section{ACKNOWLEDGMENTS}

The authors would like to thank Onyu Jung, An Chu, Thejas Wesley, Noah Lewis, Megan Jackson, Michael Pegis, Patrick Smith, and Cyrille Costentin for helpful discussions. This research was supported by the U.S. Department of Energy, Office of Science, Office of Basic Energy Sciences, under award number DE-SC0020973. C.J.K is supported by the National Science Foundation Graduate Research Fellowship under Grant No. 1122374. This research used the Inner Shell Spectroscopy 8-ID 
beamline of the National Synchrotron Light Source II, a U.S. Department of Energy (DOE) Office of Science User Facility operated for the DOE Office of Science by Brookhaven National Laboratory under Contract No. DE-SC0012704. This work made use of Shared Experimental Facilities supported in part by the MRSEC Program of the National Science Foundation under award no. DMR-1419807. Support for ICP-MS instrumentation was also provided by a core center grant P30-ES002109 from the National Institute of Environmental Health Sciences, National Institutes of Health. Y.S. acknowledges the Sloan Foundation, Research Corporation for Science Advancement (Cottrell Scholar), and the Canadian Institute for Advanced Research (CIFAR Azrieli Global Scholar).

\section{REFERENCES}

1. Murray, R. W. Chemically Modified Electrodes. Acc. Chem. Res. 1980, 13, 135-141.

2. Murray, R. W. Chemically Modified Electrodes for Electrocatalysis. Philos. Trans. R. Soc. A. 1981, 302, 253-265.

3. Bullock, R. M.; Das, A. K.; Appel, A. M. Surface Immobilization of Molecular Electrocatalysts for Energy Conversion. Chem. Eur. J. 2017, 23, 7626-7641.

4. Zhang, R.; Warren, J. J. Recent Developments in Metalloporphyrin Electrocatalysts for Reduction of Small Molecules: Strategies for Managing Electron and Proton Transfer Reactions. ChemSusChem, 2021, 14, 293-302.

5. Dalle, K. E.; Warnan, J.; Leung, J. J.; Reuillard, B.; Karmel, I. S.; Reisner, E. Electro- and Solar-Driven Fuel Synthesis with First Row Transition Metal Complexes. Chem. Rev. 2019, 119, 2752-2875.

6. Banerjee, S.; Anayah, R. I.; Gerke, C. S.; Thoi, V. S. From Molecules to Porous Materials: Integrating Discrete Electrocatalytic Active Sites into Extended Frameworks ACS Central Sci. 2020, 6, 1671-1684.

7. Berben, L.; Peters, J. C. Hydrogen evolution by cobalt tetraiminecatalysts adsorbed on electrode surfaces. Chem. Commun. 2009, 46, 398-400.

8. Canales, C.; Varas-Concha, F.; Mallouk, T. E.; Ramirez, G. Enhanced electrocatalytic hydrogen evolution reaction: Supramolecular assemblies of metalloporphyrins on glassy carbon electrodes. Appl. Catal. B Environ. 2016, 188, 169-176.

9. Thoi, V. S.; Sun, Y.; Long, J. R.; Chang, C. J. Complexes of earth-abundant metals for catalytic electrochemical hydrogen generation under aqueous conditions. Chem. Soc. Rev. 2013, 42, 2388-2400.

10. Franco, F.; Rettenmaier, C.; Jeon, H. S.; Cuenya, B. R. Transition metal-based catalysts for the electrochemical CO2 reduction: from atoms and molecules to nanostructured materials. Chem. Soc. Rev. 2020, 49, 6884-6946.

11. Manbeck, G. F.; Fujita, E. A Review of Iron and Cobalt Porphyrins, Phthalocyanines and Related Complexes for Electrochemical and Photochemical Reduction of Carbon dioxide. J. Porphyrins Phthalocyanines 2015, 19, 45-64.

12. Zagal, J. H.; Griveau, S.; Silva, J. F.; Nyokong, T.; Bedioui, F. Metallophthalocyanine-Based Molecular Materials as Catalysts for Electrochemical Reactions. Coord. Chem. Rev. 2010, 254, 2755-2791.

13. Sun, L.; Reddu, V.; Fisher, A. C.; Wang, X. Electrocatalytic reduction of carbon dioxide: opportunities with heterogeneous molecular catalysts. Energy Environ. Sci., 2020, 13, 374-403.

14. Lin, S.; Diercks, C. S.; Zhang, Y.-B.; Kornienko, N.; Nichols, E. M.; Zhao, Y.; Paris, A. R.; Kim, D.; Yang, P.; Yaghi, O. M.; Chang, C. J. Covalent organic frameworks comprising cobalt porphyrins for catalytic CO2 reduction in water. Science. 2015, 349, 1208-1213.

15. Ren, S.; Joulié, D.; Salvatore, D.; Torbensen, K.; Wang, M.; Robert, M.; Berlinguette, C. P. Molecular electrocatalysts can mediate fast, selective CO2 reduction in a flow cell. Science. 2019, 365, 367-369.

16. Liu, Y.; McCrory, C. C. L. Modulating the Electrocatalytic Mechanism of Selective CO2 Reduction by Cobalt Phthalocyanine through Polymer Coordination and Encapsulation. Nat. Commun. 2019, 10, 1683.

17. Zhanaidarova, A.; Jones, S.C.; Despagnet-Ayoub, E.; Pimentel, B.R.; Kubiak, C.P. Re(tBu-bpy)(CO) $)_{3} \mathrm{Cl}$ Supported on Multi-Walled Carbon Nanotubes Selectively Reduces $\mathrm{CO}_{2}$ in Water. J. Am. Chem. Soc., 2019, 141, 17270-17277.

18. Yao, S. A.; Ruther, R. E.; Zhang, L.; Franking, R. A.; Hamers, R. J.; Berry, J. F. Covalent Attachment of Catalyst Molecules to Conductive Diamond: $\mathrm{CO}_{2}$ Reduction Using "Smart" Electrodes. J. Am. Chem. Soc. 2012, 134, 15632-15635.

19. Hu, X.-M.; Rǿnne, M. H.; Pedersen, S. U.; Skrydstrup, T.; Daasbjerg, K. Enhanced Catalytic Activity of Cobalt Porphyrin in $\mathrm{CO} 2$ Electroreduction upon Immobilization on Carbon Materials. Angew. Chem., Int. Ed. 2017, 56, 6468- 6472.

20. Zhang, W.; Lai, W.; Cao, R. Energy-Related Small Molecule Activation Reactions: Oxygen Reduction and Hydrogen and Oxygen Evolution Reactions Catalyzed by Porphyrin- and Corrole-Based Systems. Chem. Rev. 2017, 117, 3717-3797.

21. Collman, J. P.; Devaraj, N. K.; Decréau, R. A.; Yang, Y.; Yan, Y.-L.; Ebina, W.; Eberspacher, T. A.; Chidsey, C. E. D. A Cytochrome c Oxidase Model Catalyzes Oxygen to Water Reduction Under Rate-Limiting Electron Flux. Science. 2007, $315,1565-1568$.

22. Song, E.; Shi, C.; Anson, F. C.; Comparison of the Behavior of Several Cobalt Porphyrins as Electrocatalysts for the Reduction of $\mathrm{O} 2$ at Graphite Electrodes. Langmuir. 1998, 14, 4315-4321.

23. Dey, S.; Mondal, B.; Chatterjee, S.; Rana, A.; Amanullah, S.; Dey, A. Molecular electrocatalysts for the oxygen reduction reaction. Nature Rev. Chem. 2017, 1, 0098.

24. Rigsby, M. L.; Wasylenko, D. J.; Pegis, M. L.; Mayer, J. M. Medium Effects Are as Important as Catalyst Design for Selectivity in Electrocatalytic Oxygen Reduction by Iron-Porphyrin Complexes. J. Am. Chem. Soc. 2015, 137, 4296-4299.

25. Jasinski, R. A New Fuel Cell Catalyst. Nature. 1964, 201, 1212-1213. 
26. Morozan, A.; Campidelli, S.; Filoramo, A.; Jousselme, B.; Palacin, S. Catalytic Activity of Cobalt and Iron Phthalocyanines or Porphyrins Supported on Different Carbon Nanotubes towards Oxygen Reduction Reaction. Carbon 2011, 49, 4839-4847.

27. Birdja, Y. Y.; Vos, R. E.; Wezendonk, T. A.; Jiang, L.; Kapteijn, F.; Koper, M. T. M. Effects of Substrate and Polymer Encapsulation on $\mathrm{CO}_{2}$ Electroreduction by Immobilized Indium(III) Protoporphyrin. ACS Catal. 2018, 8, 4420-4428.

28. Ge, A.; Rudshteyn, B.; Videla, P. E.; Miller, C. J.; Kubiak, C. P.; Batista, V. S.; Lian, T. Heterogenized Molecular Catalysts: Vibrational Sum-Frequency Spectroscopic, Electrochemical, and Theoretical Investigations Acc. Chem. Res. 2019, 52, 5, $1289-1300$

29. Jackson, M. N.; Surendranath, Y. Molecular Control of Heterogeneous Electrocatalysis through Graphite Conjugation. Acc. Chem. Res. 2019, 52, 3432-3441.

30. Costentin, C.; Dridi, H.; Savéant, J. M. Molecular Catalysis of $\mathrm{O}_{2}$ Reduction by Iron Porphyrins in Water: Heterogeneous versus Homogeneous Pathways. J. Am. Chem. Soc. 2015, 137, 13535-13544.

31. Kaminsky, C. J.; Wright, J.; Surendranath, Y. Graphite-Conjugation Enhances Porphyrin Electrocatalysis. ACS Catal. 2019, 9, 3667-3671.

32. Clark, M. L.; Ge, A.; Videla, P. E.; Rudshteyn, B.; Miller, C. J.; Song, J.; Batista, V. S.; Lian, T.; Kubiak, C. P. $\mathrm{CO}_{2}$ Reduction Catalysts on Gold Electrode Surfaces Influenced by Large Electric Fields. J. Am. Chem. Soc. 2018, 140, 17643- 17655.

33. Gileadi, E. Physical Electrochemistry: Fundamentals, Techniques and Applications; Wiley-VCH: Weinheim, 2011.

34. Bard, A. J. Electrochemical Methods: Fundamentals and Applications; Wiley: New York, 2001.

35. Bockris, J. O’ M.; Reddy, A. K. N.; Gamboa-Aldeco, M. Modern Electrochemistry 2A: Fundamentals of Electrodics, 2nd ed.; Kluwer Academic Publishers: New York, 2002.

36. Ryu, J, Surendranath, Y. Tracking Electrical Fields at the Pt/H2O Interface during Hydrogen Catalysis. J. Am. Chem. Soc. 2019, 141, 15524-15531.

37. Oklejas, V.; Sjostrom, C.; Harris, J. M. Surface-Enhanced Raman Scattering Based Vibrational Stark Effect as a Spatial Probe of Interfacial Electric Fields in the Diffuse Double Layer. J. Phys. Chem. B 2003, 107, 7788- 7794.

38. Ge, A.; Videla, P. E.; Lee, G. L.; Rudshteyn, B.; Song, J.; Kubiak, C. P.; Batista, V. S.; Lian, T. Interfacial Structure and Electric Field Probed by in Situ Electrochemical Vibrational Stark Effect Spectroscopy and Computational Modeling. $J$. Phys. Chem. C 2017, 121, 18674- 18682.

39. Favaro, M.; Jeong, B.; Ross, P. N.; Yano, J.; Hussain, Z.; Liu, Z.; Crumlin, E. J. Unravelling the Electrochemical Double Layer by Direct Probing of the Solid/Liquid Interface. Nat. Commun. 2016, 7 (1), 12695,

40. Fried, S. D.; Bagchi, S.; Boxer, S. G. Extreme Electric Fields Power Catalysis in the Active Site of Ketosteroid Isomerase. Science 2014, 346, 1510-1514.

41. Liu, C. T.; Layfield, J. P.; Stewart, R. J.; French, J. B.; Hanoian, P.; Asbury, J. B.; Hammes-Schiffer, S.; Benkovic, S. J. Probing the Electrostatics of Active Site Microenvironments along the Catalytic Cycle for Escherichia Coli Dihydrofolate Reductase. J. Am. Chem. Soc. 2014, 136, 10349-10360.

42. Shaik, S.; Mandal, D.; Ramanan, R. Oriented Electric Fields as Future Smart Reagents in Chemistry. Nat. Chem. 2016, 8 (12), 1091- 1098.

43. Warburton, R. E.; Hutchison, P.; Jackson, M. N.; Pegis, M. L.; Surendranath, Y.; Hammes-Schiffer, S. Interfacial FieldDriven Proton-Coupled Electron Transfer at Graphite-Conjugated Organic Acids. J. Am. Chem. Soc. 2020, 142, 2085520864.

44. Wesley, T. S.; Román-Leshkov, Y.; Surendranath, Y. ACS Cent. Sci. 2021, 7, 1045-1055.

45. Gorin, C. F.; Beh, E. S.; Bui, Q. M.; Dick, G. R.; Kanan, M. W. Interfacial Electric Field Effects on a Carbene Reaction Catalyzed by Rh Porphyrins. J. Am. Chem. Soc. 2013, 135 (30), 11257- 11265.

46. Azcarate, I.; Costentin, C.; Robert, M.; Savéant, J.-M. Through-Space Charge Interaction Substituent Effects in Molecular Catalysis Leading to the Design of the Most Efficient Catalyst of CO2-to-CO Electrochemical Conversion. J. Am. Chem. Soc. 2016, 138, 16639-16644.

47. Lau, V. M.; Gorin, C. F.; Kanan, M. W. Electrostatic Control of Regioselectivity via Ion Pairing in a Au(I)-Catalyzed Rearrangement. Chem. Sci. 2014, 5, 4975- 4979.

48. Aragonès, A. C.; Haworth, N. L.; Darwish, N.; Ciampi, S.; Bloomfield, N. J.; Wallace, G. G.; Diez-Perez, I.; Coote, M. L. Electrostatic Catalysis of a Diels-Alder Reaction. Nature 2016, 531 (7592), 88- 91

49. Kang, K.; Fuller, J.; Reath, A. H.; Ziller, J. W.; Alexandrova, A. N.; Yang, J. Y. Installation of Internal Electric Fields by Non-Redox Active Cations in Transition Metal Complexes. Chem. Sci. 2019, 10 (43), 10135- 10142

50. Chantarojsiri, T.; Ziller, J. W.; Yang, J. Y. Incorporation of Redox-Inactive Cations Promotes Iron Catalyzed Aerobic C-H Oxidation at Mild Potentials. Chem. Sci. 2018, 9 (9), 2567-2574.

51. Jackson, M. N.; Oh, S.; Kaminsky, C. J.; Chu, S. B.; Zhang, G.; Miller, J. T.; Surendranath, Y. Strong Electronic Coupling of Molecular Sites to Graphitic Electrodes via Pyrazine Conjugation. J. Am. Chem. Soc. 2018, 140, 1004-1010.

52. Jackson, M. N.; Kaminsky, C. J.; Oh, S.; Melville, J. F.; Surendranath, Y. Graphite Conjugation Eliminates Redox Intermediates in Molecular Electrocatalysis. J. Am. Chem. Soc. 2019, 141, 14160-14167.

53. Zhu, M.; Ye, R.; Jin, K.; Lazouski, N.; Manthiram, K. Elucidating the Reactivity and Mechanism of $\mathrm{CO}_{2}$ Electroreduction at Highly Dispersed Cobalt Phthalocyanine. ACS Energy Lett. 2018, 3, 1381-1386. 
54. The following three references are a brief list of studies on heterogenized porphyrins where both the amount of electroactive porphyrin and total amount of loaded porphyrin are reported for which these values do not correlate. This mismatch reveals that not all porphyrins on the surface are electroactive. While there may be many reasons for this, the most commonly invoked one is the formation of multilayers.

55. Durand, R. R.; Anson, F. C. Catalysis of dioxygen reduction at graphite electrodes by an adsorbed cobalt(ii) porphyrin. $J$. Electroanal. Chem., 1982, 134, 273-284.

56. Zhang, R.; Warren, J. J. Controlling the Oxygen Reduction Selectivity of Asymmetric Cobalt Porphyrins by Using Local Electrostatic Interactions J. Am. Chem. Soc. 2020, 142, 13426-13434.

57. Elouarzaki, K.; Le Goff, A.; Holzinger, M.; Thery, J.; Cosnier, S. Electrocatalytic Oxidation of Glucose by Rhodium Porphyrin-Functionalized MWCNT Electrodes: Application to a Fully Molecular Catalyst-Based Glucose/O2 Fuel Cell. $J$. Am. Chem. Soc. 2012, 134, 14078-14085.

58. Lennox, J. C.; Murray, R. W. Chemically modified electrodes. 10. Electron Spectroscopy for Chemical Analysis and Alternating Current Voltammetry of Glassy Carbon-Bound Tetra(aminophenyl)porphyrins. J. Am. Chem. Soc. 1978, 100, 3710-3714.

59. Rocklin, R. D.; Murray, R. W. Chemically modified carbon electrodes: Part XVII. Metallation of Immobilized Tetra(aminophenyl)porphyrin with Manganese, Iron, Cobalt, Nickel, Copper and Zinc, and Electrochemistry of Diprotonated Tetraphenylporphyrin. J. Electroanal. Chem. 1979, 100, 271-282.

60. Jester, C. P.; Rocklin, R. D.; Murray, R. W. Electron Transfer and Axial Coordination Reactions of Cobalt Tetra(Aminophenyl)Porphyrins Covalently Bonded to Carbon Electrodes. J. Electrochem. Soc. 1980, 127, 1979-1985.

61. Arrigo, R.; Hävecker, M.; Wrabetz, S.; Blume, R.; Lerch, M.; McGregor, J.; Parrott, E. P. J.; Zeitler, J. A.; Gladden, L. F.; Knop-Gericke, R.; Schlögl, R.; Su, D. S. Tuning the Acid/Base Properties of Nanocarbons by Functionalization via Amination. J. Am. Chem. Soc. 2010, 132, 9616-9630.

62. Katrib, A.; El-Egaby, M. S. The x-ray photoelectron spectroscopy of the 5-hydroxy-6-methyl-3,4-pyridinium dimethanol hexachloroplatinate(IV). Inorg. Chim. Acta, 1979, 36, L405.

63. Karweik, D. H.; Winograd, N. Nitrogen Charge Distributions in Free-Base Porphyrins, Metalloporphyrins, and their Reduced Analogs Observed by X-Ray Photoelectron Spectroscopy. Inorg. Chem. 1976, 15, 2336-2342.

64. Izutsu, K. Electrochemistry in Nonaqueous Solutions, $2^{\text {nd }}$ Ed. Wiley VCH: Germany, 2009. pp 70-71.

65. Savéant, J.-M.; Costentin, C. Elements of Molecular and Biomolecular Electrochemistry: An Electrochemical Approach to Electron Transfer Chemistry, $2^{\text {nd }}$ ed.; John Wiley \& Sons Inc.: Hoboken, NJ, 2019. pp. 209-213; 251-297.

66. Rountree, E. S.; McCarthy, B. D.; Eisenhart, T. T.; Dempsey, J. L. Evaluation of Homogeneous Electrocatalysts by Cyclic Voltammetry. Inorg. Chem. 2014, 53, 9983-10002.

67. Roberts, J. A. S.; Bullock, R. M. Direct Determination of Equilibrium Potentials for Hydrogen Oxidation/Production by Open Circuit Potential Measurements in Acetonitrile. Inorg. Chem. 2013, 52, 3823-3835.

68. Appel, A. M.; Helm, M. L. Determining the Overpotential for a Molecular Electrocatalyst. ACS Catal. 2014, 4, 630-633.

69. de Groot, M. T.; Koper, M. T. M. Redox transitions of chromium, manganese, iron, cobalt and nickel protoporphyrins in aqueous solution. Phys. Chem. Chem. Phys. 2008, 10, 1023-1031.

70. Sullivan, M. G.; Schnyder, B.; Bärtsch, M.; Alliata, D.; Barbero, C.; Imhof, R.; Kötz, R. Electrochemically Modified Glassy Carbon for Capacitor Electrodes Characterization of Thick Anodic Layers by Cyclic Voltammetry, Differential Electrochemical Mass Spectrometry, Spectroscopic Ellipsometry, X-Ray Photoelectron Spectroscopy, FTIR, and AFM. $J$. Electrochem. Soc. 2000, 147, 2636-2643

71. Atoguchi, T.; Aramata, A.; Kazusaka, A.; Enyo, M. Cobalt(II)-tetraphenylporphyrin-pyridine complex fixed on a glassy carbon electrode and its prominent catalytic activity for reduction of carbon dioxide. J. Chem. Soc., Chem. Commun. 1991, 156-157.

72. Atoguchi, T.; Aramata, A.; Kazusaka, A.; Enyo, M. Electrocatalytic activity of CoII TPP-pyridine complex modified carbon electrode for CO2 reduction. J. Electroanaly. Chem. 1991, 318, 309-320.

73. Beiler, A. M.; Khusnutdinova, D.; Wadsworth, B. L.; Moore, G. F. Cobalt Porphyrin-Polypyridyl Surface Coatings for Photoelectrosynthetic Hydrogen Production. Inorg. Chem. 2017, 56, 12178-12185.

74. See derivations in the supporting information of the following reference:

75. Jackson, M. N.; Surendranath, Y. Donor-Dependent Kinetics of Interfacial Proton-Coupled Electron Transfer. J. Am. Chem. Soc. 2016, 138, 3228-3234.

76. Fletcher, S. Tafel Slopes from First Principles. J. Solid State Electrochem. 2009, 13, 537-549.

77. Costentin, C.; Robert, M.; Savéant, J.-M. Teillout, A.-M. Concerted proton-coupled electron transfers in aquo/hydroxo/oxo metal complexes: Electrochemistry of [OsII(bpy)2py(OH2)]2+ in water. Proc. Natl. Acad. Sci, U.S.A. 2009, 106, 1182911836.

78. Costentin, C.; Robert, M.; Savéant, J.-M. Concerted Proton-Electron Transfers: Electrochemical and Related Approaches. Acc. Chem. Res. 2010, 43, 1019-1029.

79. Tyburski, R.; Liu, T.; Glover, S. D.; Hammarström, L. Proton-Coupled Electron Transfer Guidelines, Fair and Square. J. Am. Chem. Soc. 2021, 143, 560-576.

80. Irebo, T.; Zhang, M.-T.; Markle, T. F.; Scott, A. M.; Hammaström, L. Spanning Four Mechanistic Regions of Intramolecular Proton-Coupled Electron Transfer in a Ru(bpy) ${ }_{3}{ }^{2+}$-Tyrosine Complex. J. Am. Chem. Soc. 2012, 134, 16247-16254. 
81. Fukushima, T.; Drisdell, W.; Yano, J.; Surendranath, Y. Graphite-Conjugated Pyrazines as Molecularly Tunable Heterogeneous Electrocatalysts. J. Am. Chem. Soc. 2015, 137, 10926-10929.

82. Oh, S.; Gallagher, J. R.; Miller, J. T.; Surendranath, Y. Graphite-Conjugated Rhenium Catalysts for Carbon Dioxide Reduction. J. Am. Chem. Soc. 2016, 138, 1820-1823.

83. Jackson, M. N.; Pegis, M. L.; Surendranath, Y. ACS Cent. Sci. 2019, 5, 831-841.

84. Schmickler, W.; Santos, E. Interfacial Electrochemistry; Springer: Berlin, 2010, pp 51-65; 145-175.

85. See reference 35. pp. 771-894; 1068-1070.

86. See reference 65 , pp.1-28.

87. Pegis, M. L.; McKeown, B. A.; Kumar, N.; Lang, K.; Wasylenko, D. J.; Zhang, X. P.; Raugei, S.; Mayer, J. M. Homogenous Electrocatalytic Oxygen Reduction Rates Correlate with Reaction Overpotential in Acidic Organic Solutions. ACS Cent. Sci. 2016, 2, 850-856.

88. Costentin, C.; Savéant, J.-M. Towards an Intelligent Design of Molecular Electrocatalysts. Nat. Rev. Chem. 2017, 1, 0087.

89. Costentin, C.; Drouet, S.; Robert, M.; Savéant, J.-M. Turnover Numbers, Turnover Frequencies, and Overpotential in Molecular Catalysis of Electrochemical Reactions. Cyclic Voltammetry and Preparative-Scale Electrolysis. J. Am. Chem. Soc. 2012, 134 (27), 11235- 11242.

90. Hu, X.; Brunschwig, B. S.; Peters, J. C. Electrocatalytic Hydrogen Evolution at Low Overpotentials by Cobalt Macrocyclic Glyoxime and Tetraimine Complexes. J. Am. Chem. Soc. 2007, 129, 8988-8998.

91. Wakerley, D. W.; Reisner, E. Development and Understanding of Cobaloxime Activity through Electrochemical Molecular Catalyst Screening. Phys. Chem. Chem. Phys. 2014, 16, 5739- 5746.

92. Pegis, M. L.; Wise, C. F.; Koronkiewicz, B.; Mayer, J. M. Identifying and Breaking Scaling Relations in Molecular Catalysis of Electrochemical Reactions J. Am. Chem. Soc. 2017, 139, 11000-11003.

93. Klug, C. M.; Cardenas, A. J. P.; Bullock, R. M.; O'Hagan, M.; Wiedner, E. S. Reversing the tradeoff between rate and overpotential in molecular electrocatalysts for $\mathrm{H}_{2}$ production. ACS Catal. 2018, 8, 3286-3296.

94. Hoffmann, R. Solids and Surfaces: A Chemist's View of Bonding in Extended Structures; VCH Publishers, Inc.: New York, NY, 1988; pp 68-78.

95. A discussion of the complexities of adsorption to polarized interfaces can be found in reference 35, pp. 895-983.

96. Abild-Pedersen, F.; Greeley, J.; Studt, F.; Rossmeisl, J.; Munter, J. R.; Moses, P. G.; Skúlason, E.; Bligaard, T.; Nørskov, J. K. Scaling Properties of Adsorption Energies for Hydrogen-Containing Molecules on Transition-Metal Surfaces. Phys. Rev. Lett. 2007, 99, 016105.

97. Nørskov, J. K.; Bligaard, T.; Rossmeisl, J.; Christensen, C. H. Towards the computational design of solid catalysts. Nature Chem. 2009, 1, 37-46.

98. Greeley, J. Theoretical Heterogeneous Catalysis: Scaling Relationships and Computational Catalyst Design. Annu. Rev. Chem. Biomol. Eng.2016, 7, 605-635. 